\title{
Application of Precise Heat-Capacity Data to the Anal- ysis of the Temperature Intervals of the NBS (1955) and the International Practical Temperature Scales in the Region of $90^{\circ} \mathrm{K}$
}

\author{
George T. Furukawa and Martin L. Reilly
}

(September 4, 1964)

\begin{abstract}
A method is presented for analyzing the temperature intervals of the NBS (1955) temperature scale and the International Practical Temperature Scale (IPTS). Values of $d T / d R$ as a function of resistance are derived from equations of $d Q / d R$ and $d Q / d T$, obtained from precise heat-capacity data (better than \pm 0.02 percent) by the method of least squares. An adjusted temperature scale is obtained by integration of the values of $d T / d R$ relative to a reference temperature and the corresponding resistance of the thermometer. The results of the adjusting process in the neighborhood of the oxygen point $\left(90.18{ }^{\circ} \mathrm{K}\right)$ are discussed and compared with the existing temperature scales. The derived temperature scale was found to be similar to the scales maintained by the Pennsylvania State University (PSU), the Physico-Technical and Radio-Technical Measurements Institute (PRMI), and the National Physical Laboratory (NPL) from the upper limits of these scales, near $90{ }^{\circ} \mathrm{K}$, down to $50^{\circ} \mathrm{K}$. The adjusting method presented is limited by the uncertainty of formulation of $d Q / d R$ and $d Q / d T$ and the derived temperature scale is limited by the accumulated uncertainty in the integral of $d T / d R$.
\end{abstract}

\section{Introduction}

The International Practical Temperature Scale (IPTS) [1] ${ }^{1}$ is defined between -183 and $630{ }^{\circ} \mathrm{C}$ in terms of the resistance of platinum thermometers of specified characteristics, four fixed points (the triple point of water and the normal boiling points of oxygen, water, and sulfur), and two interpolation formulas (quadratic above and quartic below $0{ }^{\circ} \mathrm{C}$ ). In the region from 10 to $90^{\circ} \mathrm{K}$ a provisional temperature scale has been maintained at the National Bureau of Standards in terms of a set of platinum resistance thermometers. The thermometers now being used to maintain the NBS provisional scale are "two generations" removed from the original thermometers that were compared directly with a helium-gas thermometer in 1939 [2]. The present NBS provisional temperature scale is designated NBS (1955) and is defined to be $0.01 \mathrm{deg}$ lower than the NBS (1939) scale [2].

Since 1939 , other laboratories have also established independent temperature scales between 10 and 90 ${ }^{\circ} \mathrm{K}$, based on gas thermometry and maintained by means of platinum resistance thermometers. They are California Institute of Technology in Pasadena, Calif. [3], Pennsylvania State University in University Park, Pa. [4], Physico-Technical and RadioTechnical Measurements Institute in Moscow (U.S.S.R.) [5], and National Physical Laboratory in Teddington (U.K.) [6]. (Henceforth, the temperature scales established by the above laboratories will be referred to as CIT, PSU, PRMI, and NPL scales,

${ }_{1}$ Figures in the brackets indicate the literature references given at the end of this paper. respectively.) These temperature scales have been shown to be in agreement within $0.02 \mathrm{deg}$ over most of the 10 to $90{ }^{\circ} \mathbf{K}$ range after adjustments are made to the common oxygen-point value [3, 7].

Prior to 1939 , results of comparison of platinumresistance thermometers with a helium-gas thermometer were reported between 14 and $90{ }^{\circ} \mathrm{K}$ by Henning and Otto [8] and between 15 and $83{ }^{\circ} \mathrm{K}$ by Keesom and Bijl [9]. No accurate comparison of these temperature scales can be made with existing: scales.

Although the NBS (1955) scale was designed to join the IP'TS smoothly, the results of heat-capacity measurements made at NBS and elsewhere have consistently indicated irregularities in the region where the two temperature scales join, and in certain other regions of the NBS (1955) scale, that cannot be attributed to measurement errors. Since the heat-capacity determinations are concerned with temperature differences, any short-range variations in the "size of the degree" given by the resistance $(R)$-temperature $(T)$ relation of the thermometer can be detected. If these variations occur gradually over a broad temperature range, the present techniques used in heat-capacity determinations alone will not indicate any irregularities in the temperature scale.

A pattern of well defined irregularities has been found to exist in the relative deviations of the experimental data from an equation composed of a linear combination of Debye and Einstein heat-capacity functions which have been fitted to the data by the method of least squares. A composite deviation plot for 11 independent sets of NBS data each fitted by such an equation is shown in figure 1. (A set of 
data consists of the measurements of energy increments and the corresponding thermometer resistances from about 15 to $380^{\circ} \mathrm{K}$ obtained in the heatcapacity determinations on the empty vessel or on the vessel with sample and analyzed as a single set.) Three different calorimetric apparatus were used over the period of time in which these experimental data were obtained and a different capsule-type platinum resistance thermometer was used with each apparatus. The thermometers were calibrated at the NBS, the first ( $\mathrm{L}-18)$ in 1943, the second (769894) in 1950, and the third (1057849) in 1953. Deviation curves of the data from simpler equations, such as a single Debye heat-capacity function or a cubic equation, exhibit irregularities at the same temperatures as shown in figure 1 . The similarity of the irregularities observed in different measurements and equations used in analyzing the data indicate that the temperature scales as represented by NBS (1955)-IP'TS scales do not have uniform temperature intervals with respect to the thermodynamic temperature scale, particularly in the region of the oxygen point.
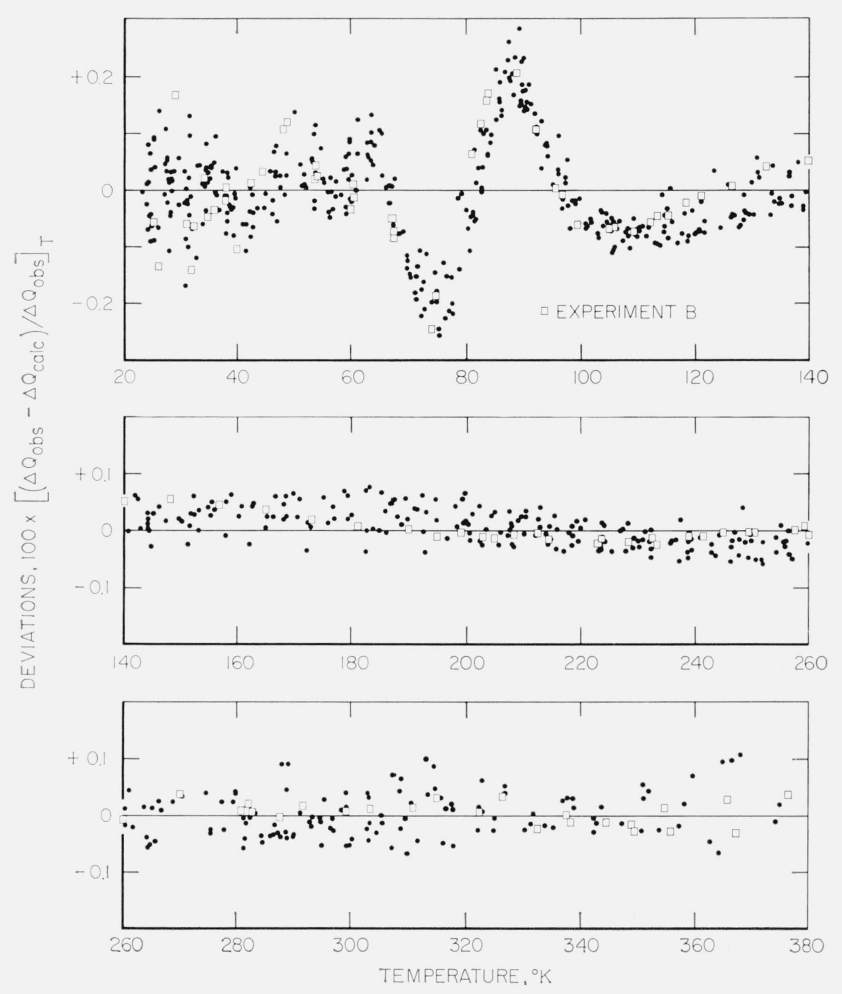

Figure 1. Composite of the deviations (percent),

$100 \times[(\Delta \mathrm{Q}$ (observed $)-\Delta \mathrm{Q}($ calculated $)) / \Delta \mathrm{Q}($ observed $)]_{\mathrm{T}}$,

for eleven (11) heat-capacity determinations in which three different thermometers ( $L-18,769894$, and 1057849) were used.

The values of $\Delta Q$ (calculated) were obtained from equations containing a series of Debye and Einstein function terms with characteristic temperatures arbitrarily selected and coefficients determined by fitting to $\Delta Q$ (observed) using the least squares method. A typical experiment (experiment $B$, used in smoothing the
temperature scale) is shown distinct from other data by open squares.
The resistance-temperature relation of the IP'TS begins to exhibit relatively large curvature below about $120^{\circ} \mathrm{K}$. The IP'TS formulation introduces an incorrect slope at the oxygen point and the IP'TS above this temperature is distorted from that of the thermodynamic temperature scale. The change in resistance with temperature at the oxygen point $\left(1 / R_{0}\right)(d R / d T)_{\mathrm{O}_{2}}$, for the IP'TS formulation is about $4.348 \times 10^{-3} / \mathrm{deg}$ for 12 thermometers in use in the low-temperature calorimetry laboratory at NBS. (In the expression $\left(1 / R_{0}\right)$ $(d R / d T)_{\mathrm{O}_{2}}, \quad R_{0}$ is the thermometer resistance at $0{ }^{\circ} \mathrm{C}$ and the subscript $\mathrm{O}_{2}$ indicates the oxygen point.) The values for $\left(1 / R_{0}\right)(d R / d T)_{\mathrm{O}_{2}}$ on the NBS (1955) scale for these thermometers are within about $\pm 0.001 \times 10^{-3} / \mathrm{deg}$ of the above value, which indicates that the scales appear to join satisfactorily at $90.18{ }^{\circ} \mathrm{K}$. The difference between the values of $\left(1 / R_{0}\right)(d R / d T)_{\mathrm{O}_{2}}$ from the gas thermometer comparison and the IPTS formulation cannot be obtained for the thermometer $(\mathrm{L}-6)$ used to represent the NBS (1939) scale [2]. The thermometer was apparently not calibrated on the IP'TS. The PRMI scale [5] is based on a group of four platinum thermometers constructed from a platinum wire designated UX-6. This scale has not been adjusted to join with the IPTS and its value for $\left(1 / R_{0}\right)(d R / d T)_{\mathrm{O}_{2}}$ is approximately $4.342 \times 10^{-3} / \mathrm{deg}$. Using the IPTS calibration constants $(\alpha=0.0039251$, $\delta=1.4899$, and $\beta=0.1127$ ) given for the UX-6 group standard [10], $\left(1 / R_{0}\right)(d R / d T)_{\mathrm{O}_{2}}$ calculates to be $4.350 \times 10^{-3} / \mathrm{deg}$, which is $0.008 \times 10^{-3} / \mathrm{deg}$ higher than the PMRI scale value. (Variations in the slope of this magnitude $(0.2$ percent $)$ are easily detected in precision calorimetry.) The resistance-temperature relation of platinum has also an inflection point at about $87^{\circ} \mathrm{K}$; the slope is, therefore, the highest at this temperature. Under these circumstances, the smooth joining of the NBS (1955) scale or any of the other scales mentioned above with the present IPTS would be expected to yield a temperature scale whose intervals are not consistent with those of the thermodynamic scale.

In the present paper the locations of the irregularities in the temperature scales are pointed out and a method is presented for adjusting the NBS (1955) scale and the IPTS, using very precise heatcapacity data.

\section{Method}

The heat capacity of a system is defined by

$$
C=d Q / d T=\lim _{\Delta T \rightarrow 0}(\Delta Q / \Delta T),
$$

so that in principle the heat capacity can be determined from precise measurements of the temperatures, $T$, and the energy increments, $\Delta Q$, introduced. Except for systems that have transitions at unique temperatures, the heat capacity should be a smooth function of the thermodynamic temperature scale. 
The resistance of platinum is also a smooth function of the temperature. (For the heat data obtained between 15 and $380^{\circ} \mathrm{K}$ on the systems under discussion and for the platinum resistance thermometer, a smooth function is defined and restricted to the following properties: (a) continuous derivatives, (b) monotonic increasing with not more than two inflection points, and (c) sigmoidal configuration of the heat-capacity curve.) The heat capacity can be expressed alternatively by

$$
C=d Q / d T=(d Q / d R)(d R / d T)
$$

which shows that the heat capacity can be calculated from corresponding values of $d Q / d R$ and $d R / d T$. The values of $d Q / d R$ can be obtained as a function of the resistance $R$ (or the temperature $T$ ) of the thermometer from observed values of $\Delta Q$ and the associated resistances. The values of $d R / d T$ corresponding to the resistance $R$ (or the temperature $T$ ) can be obtained from the calibration of the thermometer used in the measurements. The problem is, however, that the values of $d R / d T$ for the NBS (1955)_-IP'TS scales do not yield smooth, "wellbehaved" values of $d Q / d T$ expected. In addition, the accuracy of $d R / d T$ is limited in the NBS (1955) scale range because of the tabular nature of the scale. These irregularities of $d Q / d T$ will appear at the same temperatures as found in the data analyses as shown in figure 1, where the observed thermometer resistances are converted to temperatures and the values of $\Delta Q$ and $T$ analyzed.

The method presented in this paper for adjusting the temperature scales involve the relation:

$$
d T / d R=(d Q / d R) /(d Q / d T)
$$

whereby the above process (eq (2)) is reversed and values of $d T / d R$ are obtained from the smooth values of $d Q / d R$ and $d Q / d T$. Smoothed values of $d Q / d R$ are obtained as a function of the resistance $R$ of the thermometer from the observations of $\Delta Q$ 's and the corresponding resistances. Smoothed values of $d Q / d T$ are obtained in the usual manner by fitting equations to the observed $\Delta Q$ 's and temperatures, obtained by converting the thermometer resistance to temperatures. The $d Q / d T$ values from the fitted equations are taken to be correct and the irregularities in the $d Q / d T$ are disregarded because they should not exist. The values of $d T / d R$ are obtained in accordance with eq (3) as a function of the resistance from corresponding values of $d Q / d R$ and $d Q / d T$. The values of $d T / d R$ are then integrated relative to a reference temperature (for example, 90.18 or $273.15^{\circ} \mathrm{K}$ ) and the corresponding thermometer resistance to obtain an adjusted temperature scale. (Henceforth, the values of $d T / d R$ (or $d R / d T$ ) obtained from the heat-capacity data will be referred to as derived $d T / d R$. The temperature scale obtained by integration of $d T / d R$ will be described by the same adjective.)
The method has several limitations with strong dependence on accurate formulation of $d Q / d T$ as a function of the temperature. The "kinks" shown in figure 1 must be smoothed out properly without gain or loss of enthalpy change or degrees between two fixed points. A linear combination of Einstein and Debye heat-capacity functions was found from experience to yield the most suitable heat-capacity-like equation. The equations of $d Q / d T$ were analyzed by examining the higher derivatives for smoothness and the required configuration in accordance with the specifications previously given. Although the functional form of $d Q / d T$ meets the requirements set forth and is obtained by fitting the data over a broad range of temperature, other equally acceptable expressions of $d Q / d T$ may be obtained. Inasmuch as each set of data that have been analyzed contain a fairly large number of observations, it is expected that any variations in $d Q / d T$ resulting from additional observations would be smaller than the irregularities in the NBS (1955)-IP'TS scales. Also, the differences between the given equations and any other formulation of $d Q / d T$ that meets the requirements of smoothness and configuration should not exceed the deviations shown in figure 1. The shape of the functional form is very much dependent upon the distribution of the temperatures of the experimental points. At this time no investigation has been made on the most suitable distribution of experimental data. Since the energy introduced can be determined accurately, the accuracy of $d Q / d R$ was limited in the data analyzed only by the resistance measurements. Below about $40^{\circ} \mathrm{K}$ the relative accuracy of $d Q / d R$ decreased because of the small changes of resistance that were measured. The accuracy could be improved by using a thermometer of higher $d R / d T$. The values of $d Q / d R$ increase very rapidly with decrease in temperature below about $25^{\circ} \mathrm{K}$, because the thermometer resistance begins to decrease more rapidly with decrease in temperature than the heat capacity. (See insert, fig. 5.) Thus, an accurate formulation of $d Q / d R$ was not favorable below about $25^{\circ} \mathrm{K}$. The method permits smoothing of the temperature scale about a reference temperature by integration of the values of $d T / d R$, but the range of temperature is limited by the accumulation of the uncertainty in the integral of $d T / d R$.

Since the temperature intervals of the NBS (1955) - IP'TS scales are already close to those of the thermodynamic scale, the derived temperature scale obtained by integration should not deviate drastically from the original NBS-IP'TS scales as long as the values of $d T / d R$ obtained are sufficiently precise. The values of $d T / d R, d Q / d R$, and $d Q / d T$ do not change rapidly with temperature, therefore the possible uncertainty (a few hundredths of a degree) between the thermodynamic temperature scale and the NBS (1955) scale and IP'TS should not have significant effect on the integrated temperature scale. (See sec. 3.) The heat data used in the smoothing process were precise to about \pm 0.02 percent or better rom 40 to $380^{\circ} \mathrm{K}$. Below $40^{\circ} \mathrm{K}$ the data were less precise because of the decreasing sensitivity of the thermometer. 


\section{Analysis of the Heat Data and Results of the Smoothing Process}

The measured quantities in the determination of heat capacity $(C)$ are series of thermometer resistances $(R)$ or the converted temperatures $(T)$ before and after the introduction of precisely known energy increments $(\Delta Q)$. If

and

$$
C=d Q / d T=f(T)
$$

then

$$
d Q / d R=f(R)
$$

and

$$
\Delta Q=\int_{T_{1}}^{T_{2}} C d T=\int_{T_{1}}^{T_{2}} f(T) d T
$$

$$
\Delta Q=\int_{R_{1}}^{R_{2}} f(R) d R .
$$

The experimental data were analyzed in accordance with eqs (6) and (7). The function $f(T)$ was formulated as a linear combination of Debye and Einstein heat-capacity functions

$$
d Q / d T=f(T)=\sum_{i=1}^{9} a_{i} D\left(\theta_{i} / T\right)+\sum_{j=1}^{10} a_{j} E\left(\omega_{j} / T\right)
$$

with the characteristic temperature for each term arbitrarily selected and the corresponding coefficient determined by the method of least squares from the observed data. The characteristic temperatures used were: $\theta_{i}=50,100,150,200,250,300,400,500$, and 700 and $\omega_{j}=100,150,200,250,300,400,500$, 700,900 , and 1100 . The function $f(R)$ was obtained as smoothed tabular values at regularly spaced intervals of resistance. The table was based on three partially overlapping polynomials of the form

$$
d Q / d R=f(R)=\sum_{n=-4}^{4} a_{n} R^{n},
$$

fitted to the data by the method of least squares. A high speed digital computer at the NBS was used in the calculations.

The heat-capacity data from six different sets of measurements that were obtained recently and considered to be the most precise were analyzed. These data are part of those shown in the composite plot of figure 1. The same capsule-type thermometer No. $769894, \quad\left(\alpha=0.00392536, \delta=1.49_{30}, \quad \beta=0.11014\right.$, and $R_{0}=25.54772 \mathrm{ohms}$ ) was used with the same calorimeter vessel that was fabricated principally of copper and assembled with "silver solder" and tinlead solder. The inner surface of the vessel was covered with pure tin and the outer surface gold plated. (For details of the calorimeter design and measurement procedures, see references [11, 12].) Of these data, three sets were from completely independent measurements on the empty vessel and the other three sets were from measurements on the vessel containing samples. The measurements on the empty vessel were made immediately after each measurement with the sample. Henceforth, these sets of experiments will be identified alphabetically, the capital letter representing the vessel-plus-sample measurements and the lower case letter the corresponding empty-vessel measurements. The letter $A$ corresponds to the measurements obtained with $\mathrm{BeO} \cdot \mathrm{Al}_{2} \mathrm{O}_{3}, B$ with $\mathrm{Al}_{4} \mathrm{C}_{3}$, and $C$ with $\mathrm{LiAlH}_{4}$. The functions $f(T)$ and $f(R)$ were obtained over the complete temperature range of each set of measurements (fig. 1). The analysis of the calorimeter vessel-plus-sample data was complicated since the heat capacity of the sample was superimposed on that of the vessel. Deviations (percent),

$$
100 \times\left[\left(\Delta Q_{\mathrm{obs}}-\Delta Q_{\mathrm{calc}}\right) / \Delta Q_{\mathrm{obs}}\right]_{T}
$$

of each set of measurements from their respective functions $f(T)$ are shown in figure 2 . The corresponding deviations,

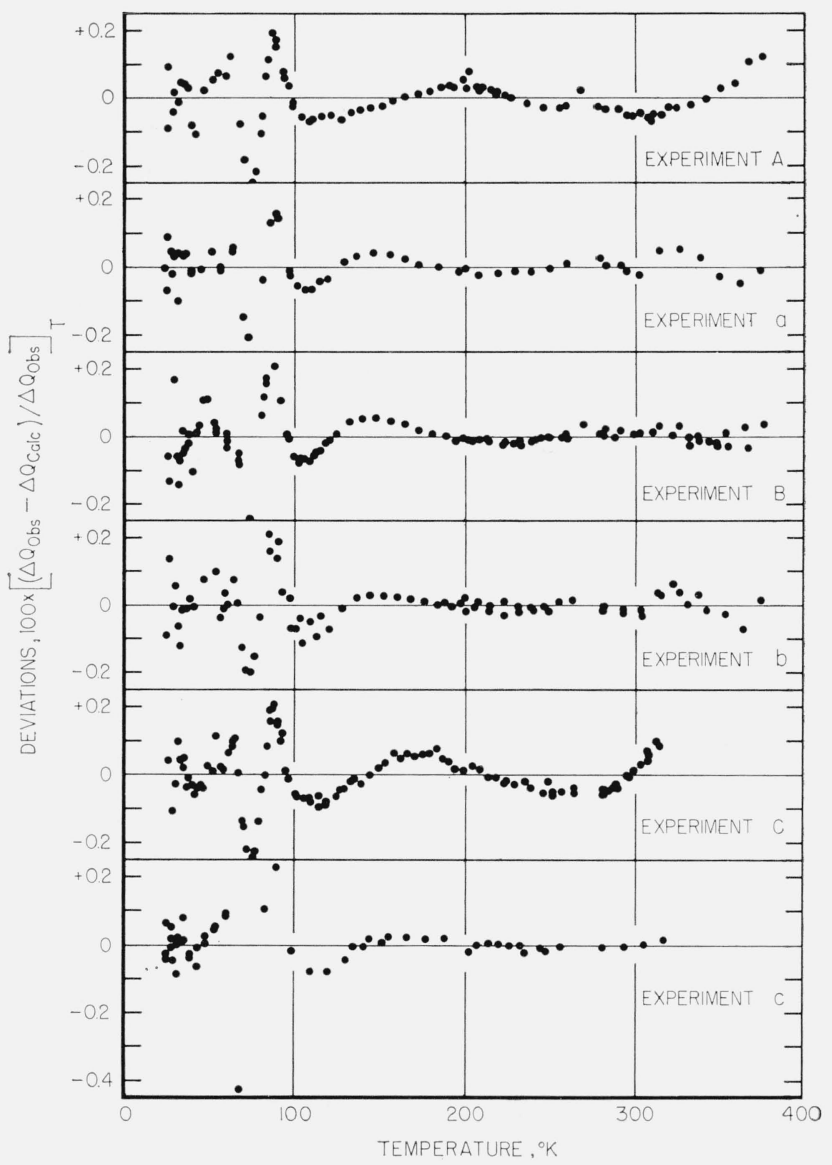

Figure 2. The deviations (percent),

$100 \times[(\Delta \mathrm{Q}$ (observed $)-\Delta \mathrm{Q}($ calculated $)) / \Delta \mathrm{Q}($ observed $)]_{\mathrm{T}}$,

for each of the six (6) sets of measurements obtained using thermometer No.769894.

These were the measurements analyzed to smooth the NBS (1955)-IPTS scales. The values of $\Delta Q$ (calculated) were obtained from $f(T)$ at the appropriate experimental temperatures. 


$$
100 \times\left[\left(\Delta Q_{\mathrm{obs}}-\Delta Q_{\mathrm{calc}}\right) / \Delta Q_{\mathrm{obs}}\right]_{R}
$$

of these same sets of measurements from functions $f(R)$ are shown in figure 3 . The subscripts $T$ and $R$ indicate the methods (eqs (6) and (7), respectively) by which $\Delta Q$ (calculated) was obtained.

In figures 4 and 5 are shown the plots of $f(T)$ and $f(R)$ for the data analysed. The three measurements of the empty vessel $(a, b, c)$ are coincident on the scale shown in the plots. The plots are given to show the broad range of magnitudes of values of $d Q / d T$ and $d Q / d R$ of each set of data. The magnitudes of $d Q / d T$ and $d Q / d R$ of the different sets of measurements are sufficiently diverse to permit detecting whether or not an irregular characteristic or "kink" common to these sets of measurements is additive. In particular, any "kink" in the heatcapacity curve of the empty vessel should be exhibited in figures 1 and 2 as a regular pattern of relative deviation inversely proportional in magnitude to the magnitude of $d Q / d T$. The deviations of figures 1 and 2 disclose regular proportional errors of about the same magnitude. Calibration errors in any bridge or potentiometer settings will be revealed as a fixed pattern in the relative deviation

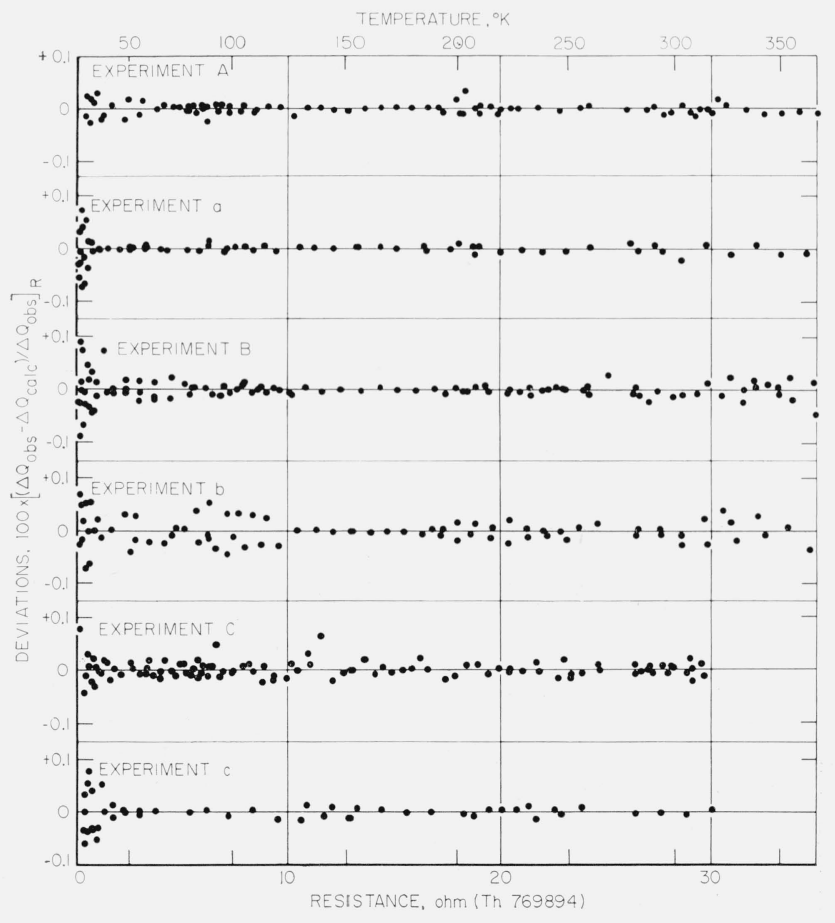

Figure 3. The deviations (percent),

$100 \times[(\Delta \mathrm{Q}$ (observed $)-\Delta \mathrm{Q}($ calculated $)) / \Delta \mathrm{Q}($ observed $)]_{\mathrm{R}}$,

for each of the six (6) sets of measurements obtained using thermometer No. 769894 .

These were the measurements analyzed to smooth the NBS (1955)-IPTS scales. The values of $\Delta Q$ (calculated) were obtained from $f(R)$ at the appropriate experimentally observed resistances. recurring whenever that dial position is used throughout the temperature range of measurements. The lack of a repeating irregularity in the deviation plots of figures 1 and 2 and the lack of repeating or large irregularities in the deviation plots of the analysis of $\Delta Q$ versus $R$ of figure 3 discount the existence of any such large calibration errors in the above instruments.

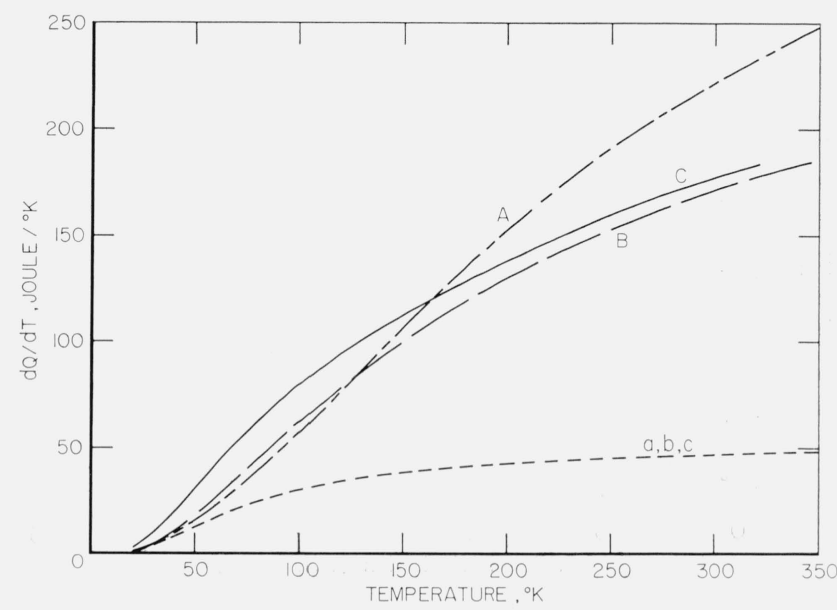

Figure 4. Comparison of the smoothed heat capacities $\mathrm{f}(\mathrm{T})$ of experiments used in smoothing the NBS (1955)-IPTS scales.

The heat capacities of the empty vessel (experiments $a, b$, and $c$ ) are virtually coincident.

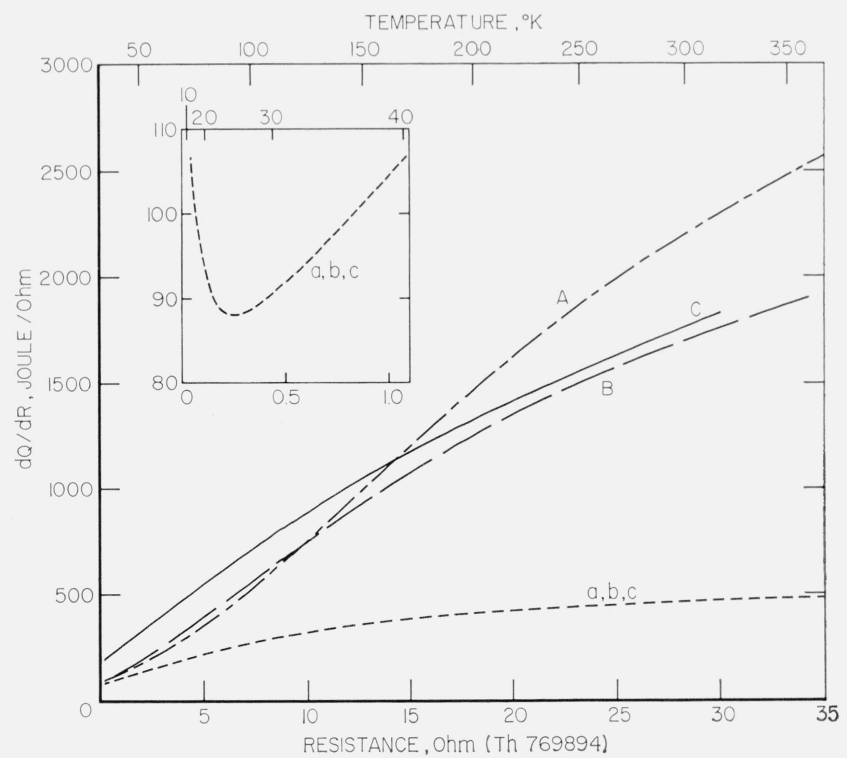

Figure 5. Comparison of the values of $\mathrm{f}(\mathrm{R})$ of experiments used in smoothing the NBS (1955)-IPTS scales.

The values of $f(R)$ of the empty vessel (experiments $a, b$, and $c$ ) are virtually coincident. 


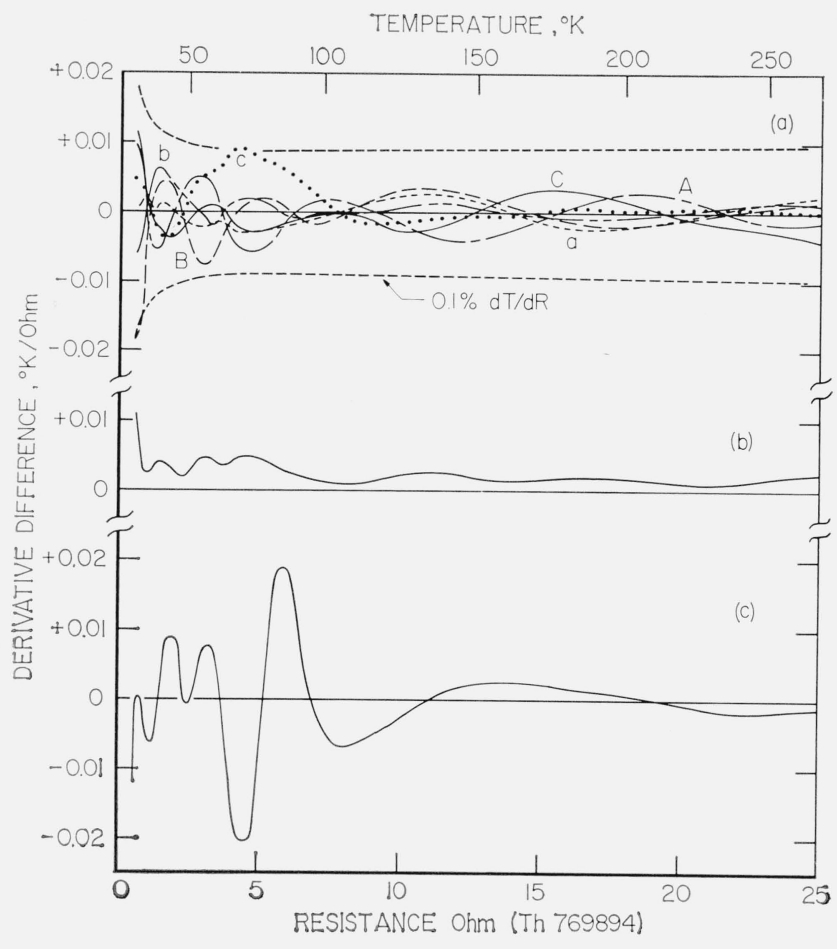

Figure 6. (a) Deviation from their mean of $\mathrm{dT} / \mathrm{dR}$ determined from each of the six experimental measurements.

(b) Root-mean-square deviation from the mean of the six sets of $\mathrm{dT} / \mathrm{dR}$.

(c) Difference in $\mathrm{dT} / \mathrm{dR}$ between derived scale and NBS (1955)IPTS scales (base line).

Figure 6 a shows the deviations of the $d T / d R$ obtained from each set of measurements from their mean. The root-mean-square (rms) deviation from their mean plotted in figure $6 \mathrm{~b}$ shows that the agreement in the derived $d T / d R$ is best in the neighborhood of the oxygen point. The rms deviation increases to about 0.02 percent at temperatures above and 0.04 percent below the oxygen point, respectively. The deviation of the mean derived $d T / d R$ from the $d T / d R$ of the NBS (1955)-IP'TS scales shown in figure $6 \mathrm{c}$ is similar to the

$$
100 \times\left[\left(\Delta Q_{\text {obs }}-\Delta Q_{\text {calc }}\right) / \Delta Q_{\text {obs }}\right]_{T}
$$

plots shown in figures 1 and 2. The apparently high (or low) values shown in figures 1 and 2 of $\Delta Q$ (observed) over those of $\Delta Q$ (calculated) arise from values of $\Delta T$ that are too small (or too large) corresponding to the observed $\Delta R$. Since the $\Delta T$ corresponding to the $\Delta R$ has been adjusted accordingly in the derived values of $d T / d R$, the configuration of figures 1,2 , and 6 a should be very similar.

In figure 7 the mean derived $d R / d T$ (reciprocal of $d T / d R$ (obtained) is compared with those of the NBS (1955)-IPTS scales in the neighborhood of the oxygen point. The shape of the derived $d R / d T$ is considerably different from that of the NBS (1955)IPTS scales. The inflection point at $90.18^{\circ} \mathrm{K}$ of the $d T / d R$ of the NBS (1955)-IPTS scales has been displaced in the derived $d T / d R$ to around $110{ }^{\circ} \mathrm{K}$. This should yield a temperature scale more compatible with the heat-capacity measurements. The general configurational relation between the curves in the interval 75 to about $95^{\circ} \mathrm{K}$ is very similar to that reported by Borovick-Romanov [5] for the PRMI and NBS (1955) scales and the IPTS using UX-3 and UX-6 platinum thermometers.

Figure 8 a shows between 30 and $220{ }^{\circ} \mathrm{K}$ the deviation of the temperatures derived by integration of $d T / d R$ relative to the oxygen point of each set of measurements from their mean. The rms deviation of the derived temperature scales given in figure $8 \mathrm{~b}$ shows that the agreement is best between about 60 and $150{ }^{\circ} \mathrm{K}$. Beyond these temperatures the rms deviation is about $0.01 \mathrm{deg}$. The divergence below the oxygen point of the temperature scale derived from experiment $c$ is shown to be unusually high. This is considered to be due to the temperatures at which the experimental data were taken.

Figure $8 \mathrm{c}$ shows the deviation of the mean derived temperature scale from NBS (1955)-IP'TS scales. The average of the deviations of the PSU, PRMI, and NPL scales from the NBS (1955) scale is also shown.

As a part of the program of the International Committee on Weights and Measures, the National Physical Laboratory and the Physico-Technical and Radio-Technical Measurements Institute intercompared platinum resistance thermometers that were calibrated on the NBS (1955), PSU, PRMI, and NPL temperature scales. These data made available to us through J. L. Riddle (NBS), were used freely in comparing them with the derived temperature scale. Both the NPL data and the PRMI data on the resistance thermometers representing each of the scales were assumed to have been observed at the same temperatures. For convenience of the subsequent discussion the PSU, PRMI, and NPL temperature scales will be referred to collectively as non-NBS temperature scales. The derived temperature scale was found to track the average of the non-

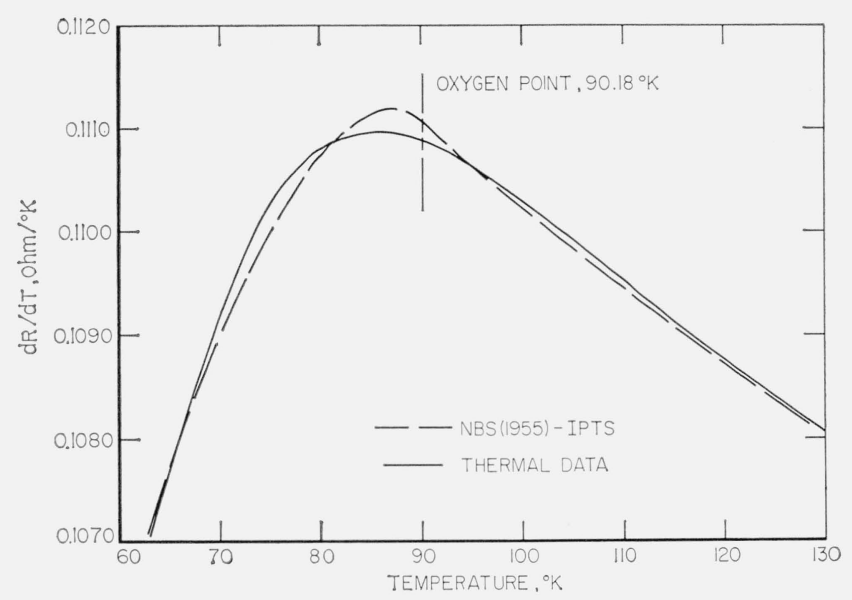

Figure 7. Comparison of the derived values of $\mathrm{dR} / \mathrm{dT}$ with those of the NBS (1955)-IPTS scales. 


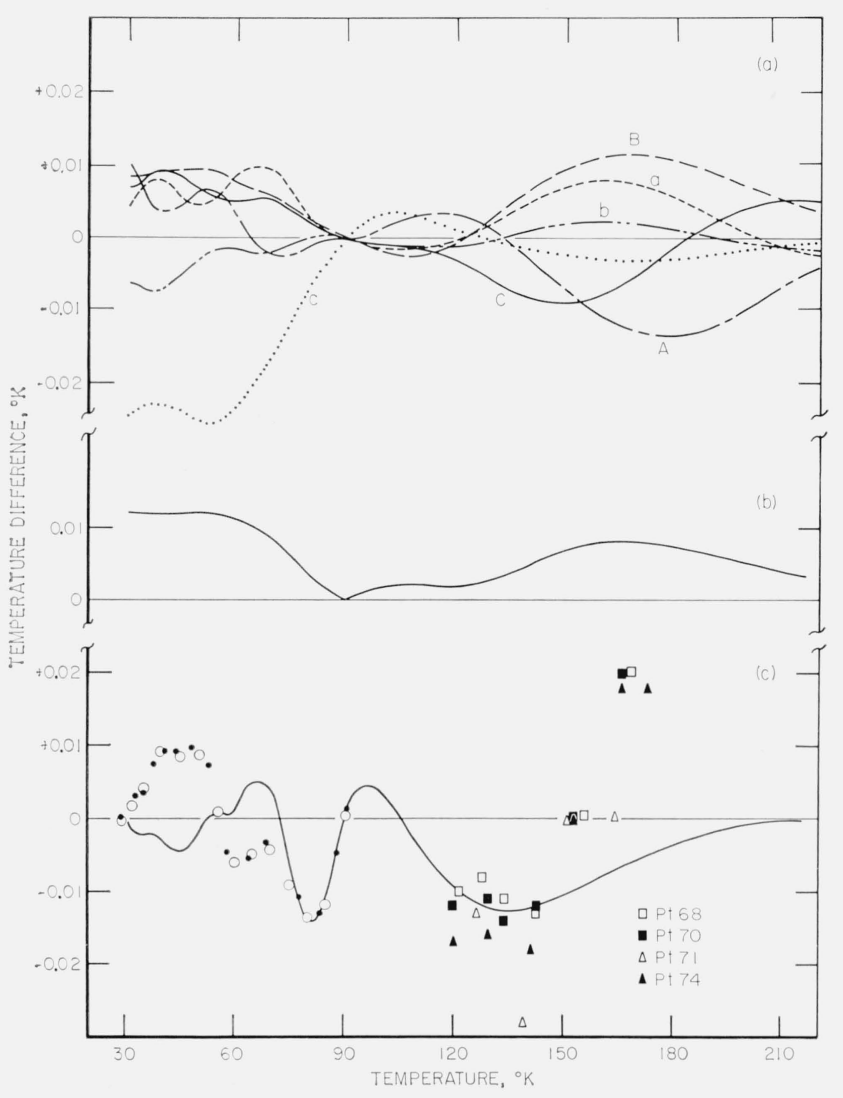

Figure 8. (a) Deviations from their mean of the temperature scales derived from each of the six (6) experiments.

The temperature scales were obtained by integration relative to the oxygen point $\left(90.18^{\circ} \mathrm{K}\right)$.

(b) Root-mean-square deviation from their mean of the six derived temperature scales.

(c) Deviations from the NBS (1955) - IPTS scales of the mean derived temperature scale.

The symbols $\bigcirc$ and indicate, respectively, the mean of the deviations of the PSU, PR MI, and NPL temperature scales from the NBS (1955) scale as observed at NPL and PRMI. The symbols $\square, \boldsymbol{a}, \triangle$, and $\boldsymbol{\Lambda}$ indicate deviations from the IPTS of the helium-gas thermometer reported by Keesom and Dammers [13] for platinum thermometers designated $\mathrm{Pt} 68,70,71$, and 74 , respectively.

NBS scales closely from the oxygen point down to about $75^{\circ} \mathrm{K}$. From 75 down to about $55^{\circ} \mathrm{K}$ the configuration is similar, but the maximum deviation is about $0.01 \mathrm{deg}$. Below $55^{\circ} \mathrm{K}$, the shape is different and the deviation is as large as $0.014 \mathrm{deg}$.

In the region above the oxygen point, a portion of the data on the intercomparison of the IPTS with a helium-gas thermometer obtained by Keesom and Dammers [13] is plotted in figure 8c. In the region of about 120 to $140{ }^{\circ} \mathrm{K}$, these data are in fair agreement with the derived scale. Above about $140^{\circ} \mathrm{K}$, there is considerable divergence.

The deviations shown below $55^{\circ} \mathrm{K}$ and above $140{ }^{\circ} \mathrm{K}$ in the comparisons given in figure $8 \mathrm{c}$ are considered to be due to the inability of the method presented to account for any long-range, gradual variations in the NBS (1955)-IPTS scales. Therefore, the deviations of the observations shown in $8 \mathrm{c}$ are not indicative of any variations in the data.

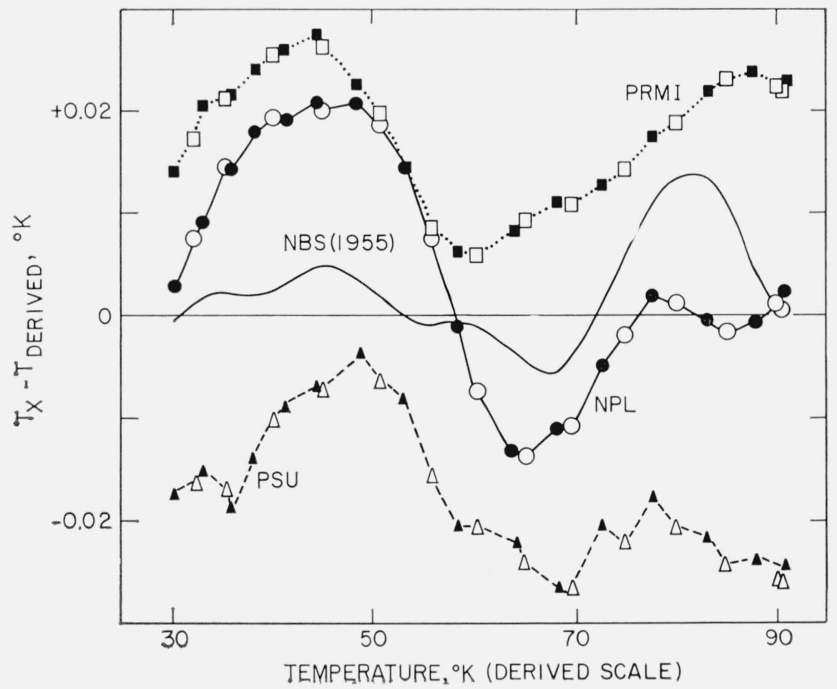

Figure 9. Deviations of the NBS (1955), PSU, PRMI, and NPL temperature scales from the derived temperature scale.

The open and filled symbols indicate, respectively, the data obtained at NPL and PRMI.

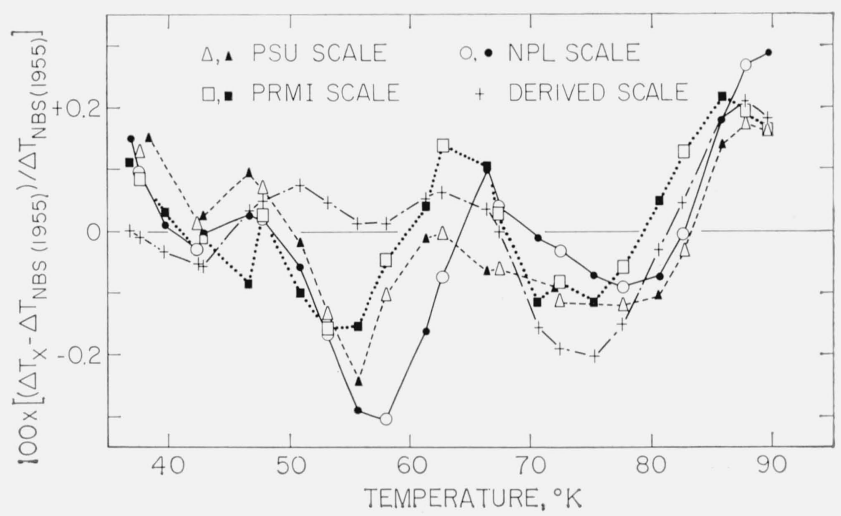

Figure 10. Deviations (percent),

$$
100 \times\left[\left(\triangle T_{x}-\triangle T_{N B S(195 \delta)}\right) / \triangle T_{N B S(195 \tilde{)})}\right],
$$

of PSU, PRMI, NPL, and the derived temperature scales.

The subscript $x$ indicates any of the above temperature scales. The values of $\triangle T$ were obtained from the same data obtained at the NPL and PRMI for the comparison of the NBS (1955), PSU, PRMI, and NPL temperature scales. The abscissa for the points plotted is the mean temperature of the two temperature used to obtain $\triangle T$.

The deviations of the NBS (1955) and the nonNBS temperature scales from the derived scale are shown from 30 to $90{ }^{\circ} \mathrm{K}$ in figure 9 . Except for the displacement of the scales, due to the use of numerically different values for the oxygen point, the PSU scale is in excellent agreement with the derived scale from 90 down to about $55^{\circ} \mathrm{K}$, the NPL scale down to $70^{\circ} \mathrm{K}$, and the PRMI scale down to $75^{\circ} \mathrm{K}$. The NBS (1955) scale is shown to be relatively divergent from each of the non-NBS temperature scales. The NBS (1955) and the derived scale are in good agreement below $70^{\circ} \mathrm{K}$.

A comparison of the "size of the degree", or effectively the values of $d T / d R$ used to obtain the derived temperature scale, is made with those of other scales in figure 10 . The differences in the 
successive temperatures obtained for the various temperature scales are compared with the differences in the corresponding successive temperatures on the NBS (1955) scale by the relation:

$$
100 \times\left[\left(\Delta T_{x}-\Delta T_{\mathrm{NBS}}\right) / \Delta T_{\mathrm{NBS}}\right],
$$

where the subscript $x$ represents any of the other temperature scales. The configuration of the relation given by eq (10) for the derived scale is similar to those of the non-NBS scales over the entire temperature range shown in figure 10 . This similarity indicates that the derived and non-NBS temperature scales are closely alike. It is remarkable that the derived temperature scale agrees closely with the non-NBS scales.

\section{Conclusions}

The temperature intervals of the NBS (1955) IP'TS scales were adjusted, utilizing values of $d T / d R$ that were derived from six independent sets of heatcapacity data. These data were fitted by the method of least squares to equations representing $d Q / d T$ and $d Q / d R$ which differ over a broad range in the magnitude of their values. The rms deviations of the values of $d T / d R$ obtained from the six sets of data are from 0.01 to 0.02 percent above $90^{\circ} \mathrm{K}$ and increase to about 0.04 percent at $70^{\circ} \mathrm{K}$ and lower. Comparison of the derived temperature scale, obtained by integrating the values of $d T / d R$ relative to the oxygen point, with the mean of the non-NBS temperature scales indicates agreement to better than $0.01 \mathrm{deg}$ from 90 down to about $55^{\circ} \mathrm{K}$ with slightly larger deviations at lower temperatures and falls well within the range of the existing scales. Between 60 to $90{ }^{\circ} \mathrm{K}$, the deviation of the "size of the degree" of the derived scale from the mean value of the non-NBS scales is comparable or smaller than the spread of the values of the non-NBS scales. Below $60^{\circ} \mathrm{K}$, slightly larger deviations were obtained.

Because of the lack of data, no accurate comparison can be made of the derived temperature scale above the oxygen point.

The method described for adjusting the temperature scales using precise heat-capacity data is limited to a short range near the reference temperature because of the accumulation of the uncertainty in the integral of $d T / d R$. An accurate formulation of the values of $d Q / d R$ and $d Q / d T$ is essential to the success of the method. A large number of properly distributed, highly precise heat-capacity data is a prerequisite. Accurate formulation of $d Q / d R$ is complicated below about $25^{\circ} \mathrm{K}$ because of its rapid increase with decrease in temperature. The relative precision at the lower temperatures would be better if a thermometer of higher $d R / d T$ is used.

A method for adjusting the temperature scale is demonstrated in this paper using the laboratory data that had accumulated in the past few years. The heat-capacity data from the measurements of the vessel plus sample had complicated shapes because of the superposition of the heat capacity of the sample on that of the vessel. Systematic measurements on systems having values of $d Q / d T$ with a simple shape should yield more accurate equations for the values of $d Q / d T$.

\section{References}

[1] H. F. Stimson, J. Res. NBS 65A, 139 (1961)

[2] H. J. Hoge and F. G. Brickwedde, J. Res. NBS 22, 351 (1939).

[3] D. M. Yost, C. S. Garner, D. W. Osborne, T. R. Rubin, and H. Russell, Jr., J. Am. Chem. Soc. 63, 3488 (1941).

[4] G. W. Moessen, J. G. Aston, and R. G. Ascah, Temperature, Its Measurement and Control in Science and Industry, Vol. 3, pt 1, p. 91 (Reinhold Publishing Corp., New York, 1962).

[5] A. S. Borovick-Romanov, P. G. Strelkov, M. P. Orlova and D. N. Astrov, Temperature, Its Measurement and Control in Science and Industry, Vol. 3, pt 1, p. 113 (Reinhold Publishing Corp., New York, 1962).

[6] C. R. Barber, Brit. J. Appl. Phys. 13, 235 (1962).

[7] C. R. Barber, Temperature, Its Measurement and Control in Science and Industry, Vol. 3, pt. 1, p. 345 (Reinhold Publishing Corp., New York, 1962).

[8] F. Henning and J. Otto, Physik. Zeitschr. 37, 601 (1936).

[9] W. H. Keesom and A. Bijl, Physica 3, 418 (1936).

[10] D. I. Sharevskaya, P. G. Strelkov, A. S. BorovikRomanov, D. N. Astrov, and M. P. Orlova, Temperature, Its Measurement and Control in Science and Industry, Vol. 3, pt. 1, p. 351 (Reinhold Publishing Corp., New York, 1962).

[11] G. T. Furukawa, M. L. Reilly, and J. H. Piccirelli, J. Res. NBS 68A, 381 (1964).

[12] A. R. Glasgow, Jr., G. S. Ross, A. T. Horton, D. Enagonio, H. D. Dixon, and C. P. Saylor; and G. T. Furukawa, M. L. Reilly, and J. M. Henning, Anal. Chem. Acta 17, 54 (1957).

[13] W. H. Keesom and B. G. Dammers, Physica 2, 1080 (1935).

(Paper 69A1-321) 